\title{
Topographically induced self-deformation of the nuclei of cells: dependence on cell type and proposed mechanisms
}

\author{
Patricia M. Davidson • Olivia Fromigué • \\ Pierre J. Marie • Vasif Hasirci · Günter Reiter • \\ Karine Anselme
}

Received: 30 June 2009/ Accepted: 21 November 2009/Published online: 10 December 2009

(C) Springer Science+Business Media, LLC 2009

\begin{abstract}
Osteosarcoma-derived cell lines (SaOs-2, MG63) have recently been shown to deform their nucleus considerably in response to surface topography. Such a deformation had not been described previously. Here we present results on additional cell lines, including cancerous (OHS4, U2OS), immortalized (F/STRO- $1^{+} \mathrm{A}$ and FHSO6) and healthy cells (HOP). The cancerous cells were found to deform extensively, the immortalized cells showed small deformations, whereas the healthy cells showed deformation only at short incubation times. These results suggest a strong link between the malignant transformation of cells and the state of the cytoskeletal network. We propose mechanisms to explain the deformation in which the cytoskeleton either pushes down on the nucleus during spreading or pulls it down upon adhesion to the pillars.
\end{abstract}

P. M. Davidson · K. Anselme $(\bowtie)$

Institut de Science des Materiaux de Mulhouse, CNRS LRC

7228, Université de Haute Alsace, 68057 Mulhouse cedex,

France

e-mail: karine.anselme@uha.fr; patricia.davidson@uha.fr

O. Fromigué · P. J. Marie

INSERM U606, Hôpital Lariboisière, 75475 Paris Cedex 10, France

O. Fromigué · P. J. Marie

Université Paris Diderot, Paris, France

V. Hasirci

METU, BIOMAT, Department of Biological Sciences,

Biotechnology Research Unit, 06531 Ankara, Turkey

G. Reiter

Albert-Ludwigs-Universität, Physikalisches Institut,

Hermann-Herder-Str. 3, 79104 Freiburg, Germany

\section{Introduction}

When a cell adheres to a substrate, the surface properties of this substrate will have a large impact on the fate of the cell. If the surface is favorable to cell attachment, it will respond by undergoing several processes. First the cell will form attachment points and the cytoskeleton will initiate spreading on the surface. On a flat surface the cell will go from a spherical shape to a flattened one. On surfaces with very large features the cell will follow the contours of the surface topography. At the micron scale, surface features are on the scale of sub-cellular components such as the nucleus. At this size scale several studies have shown that cells span the surface structures [1]. Many researchers have used flexible micropillars to study the forces generated by the cytoskeleton [2]. In one paper by Steinberg et al. [3], deformation of the nucleus of immortalized keratinocytes on micropillars can be seen but has not been discussed. Studies on the effect of microtopography on the cytoskeleton have concentrated on chemical patterning on the micron scale [4]. In these the topography served as "pinning points" to which the cell attached and was used to study the arrangement of the cytoskeleton in confined cells [5]. Preferential directionality of actin fibers has also been seen in cells grown on grooved surfaces $[6,7]$. In one of these studies the nucleus was also found to elongate in the direction of the grooves [8]. Yet, extensive deformation of the nucleus has not been described in the literature, except by us.

Recently we have shown that when the surface features have a size that is similar to the size of the organelles, osteosarcoma cells are able to conform to surface structures when grown on micropillared PLLA surfaces [9]. This deformation also extended to the interior of the cell, resulting in a deformation of the 
nucleus. This was confirmed by staining of the lamins in the nuclear membrane and observation by confocal microscopy, showing that the nucleus followed the shape of the underlying pillars [9]. This gives rise to questions regarding the architecture of the cell and possible effects on cell performance: changes to the cytoskeleton scaffolding are believed to be able to regulate cellular responses to the environment [10]. Hence, deformation of the cell, and in particular the nucleus, should have a profound impact on the functioning of the cell: it has been shown that gene expression is affected by the position of chromosomes in the nucleus and deformation would lead to unusual nucleus architecture [11]. In fact, positioning of genes near the nuclear membrane has been linked to the switching off of gene expression [12]. Extensive deformation of the nucleus increases the surface-to-volume ratio and should therefore result in a higher proportion of genes at the surface, which would result in modifications in gene expression. However, in our previous study we did not observe any influence of this deformation on the viability, proliferation and differentiation of cells, as measured by alkaline phosphatase production [9]. We also found that this deformation happened in osteosarcoma-derived cells (SaOs-2 and MG63), but very rarely in human primary osteoprogenitor cells from the bone marrow, demonstrating the differences in properties of these different types of bone cells.

It is well known that cancerous cells have mechanical properties that are different from healthy cells [13]. They have been shown to be more deformable than healthy cells and produce less cytoskeletal filaments [13]. In vitro, healthy cells can be modified with viral oncogenes to become "immortalized". This process generally involves inactivation of tumour suppressor genes, which results in increased proliferation [14]. Although these cells have now acquired a modification that is specific to cancerous cells they are often not tumorigenic and are used as a substitute for healthy cells [3]. On the other hand, studies have used immortalized cells as models for malignant cells that can be used for comparison to the healthy cells they are derived from. These have demonstrated an increase in deformability and a decrease in cytoskeletal filament production in immortalized cells [13, 15].

Following previous results obtained on cancerous and healthy cells we conducted a more thorough study on bone cells of different malignancies to understand the effect of mutations on the cell cytoskeleton. Here we present results obtained on healthy, immortalized and cancerous bone cells. It was found that the deformation of cancerous cells is extensive and universal across four osteosarcoma cell lines. In immortalized cell lines the deformation is either very weak in FHSO6 cells or only occurs at the edge of the cells in $\mathrm{F} / \mathrm{STRO}^{+} \mathrm{A}$ cells. In healthy osteoprogenitor cells the deformation occurs but only at short incubation times and diminishes rapidly. Mechanisms are proposed to explain the deformations obtained. Additionally, the timedependency of the deformation in osteoprogenitor cells and an osteosarcoma-derived cell line is used to discuss the early stages of cell adhesion on these surfaces.

\section{Materials and methods}

\subsection{Substrate preparation}

The micropillared surfaces were prepared as described previously [9]. Briefly, a film of PLLA was made by air drying a solution of PLLA in dichloromethane. This film was then placed on a hot plate and pressed with the desired PDMS stamp. The film was then cooled quickly in cold water, and removed from the stamp. The square pillars obtained were $7 \mu \mathrm{m}$ wide, had a spacing of $7 \mu \mathrm{m}$ and were $4 \mu \mathrm{m}$ tall. Prior to cell inoculation the surface was sterilized briefly in ethanol and rinsed with sterile water.

\subsection{Cell culture}

Five cells lines were used in this study. HOP cells were obtained as described previously by Anselme et al. [16]. F/STRO ${ }^{+}{ }^{+}$and FHSO6 cells were prepared as described in previous studies $[17,18]$. SaOs-2 cells were purchased from the ECACC, U2OS from the ATCC and OHS4 were provided by Rodan [19]. All cells were cultured in Iscove complete medium except for SaOs-2 cells which were cultured in McCoy complete medium, containing, in both cases, fetal bovine serum $(10 \%)$, glutamine $(2 \mathrm{mM})$, penicillin (100 units $/ \mathrm{ml})$ and streptomycin $(0.1 \mathrm{mg} / \mathrm{ml})$. Cells were inoculated on the sterilized samples at a density of 20,000 cells/well in 24-well plates (approx. 10,000 cells/ $\mathrm{cm}^{2}$ ). Culture times on the samples were 24, 48, 96 and $168 \mathrm{~h}$ for the cancerous and immortalized cells; 6, 12, 24, 48 and $96 \mathrm{~h}$ for the HOP cells and SaOs-2.

\subsection{Fluorescent labeling and imaging}

Samples were fixed and labeled as described previously [9]. Briefly, the samples were fixed with paraformaldehyde, permeabilized with Triton-X100, followed by saturation with Bovine serum albumin (1\% in PBS) and labeling with Phalloidin-FITC and DAPI. The samples were then mounted in a 1:1 solution of glycerol in PBS without antifade reagent and imaged on an Olympus BX51 epifluorescence microscope. 


\section{Results}

\subsection{Immortalized and cancerous cells}

Two lines of immortalized cells and three lines of cancerous cells (osteosarcoma) that originated from bone were grown on micropillared surfaces. The cell lines were chosen for their size which is comparable to $\mathrm{SaOs}-2$, on which previous studies had been conducted [9] (see Fig. 1).

The immunofluorescence images of the cancerous cell lines showed a high level of nucleus deformation (Fig. 2). This is accordance with previously reported data on MG-63 and SaOs-2 [9]. In all cases the cytoskeleton and the nucleus were both extensively deformed and the position of
Fig. 1 Size and shape comparison of the cell lines described in this study. Six cell lines were used in this study including one healthy cell line (HOP) two immortalized cell lines (F/STRO $-1^{+} \mathrm{A}$ and FHSO6) and three cancerous cell lines (SaOs-2, OHS4 and U2OS). HOP cells are larger and more elongated. F/STRO$1^{+} \mathrm{A}$ cells are of comparable size to the others, but also FHSO6 and the three cancerous cell lines all have similar sizes and morphology elongated with long filopodia.
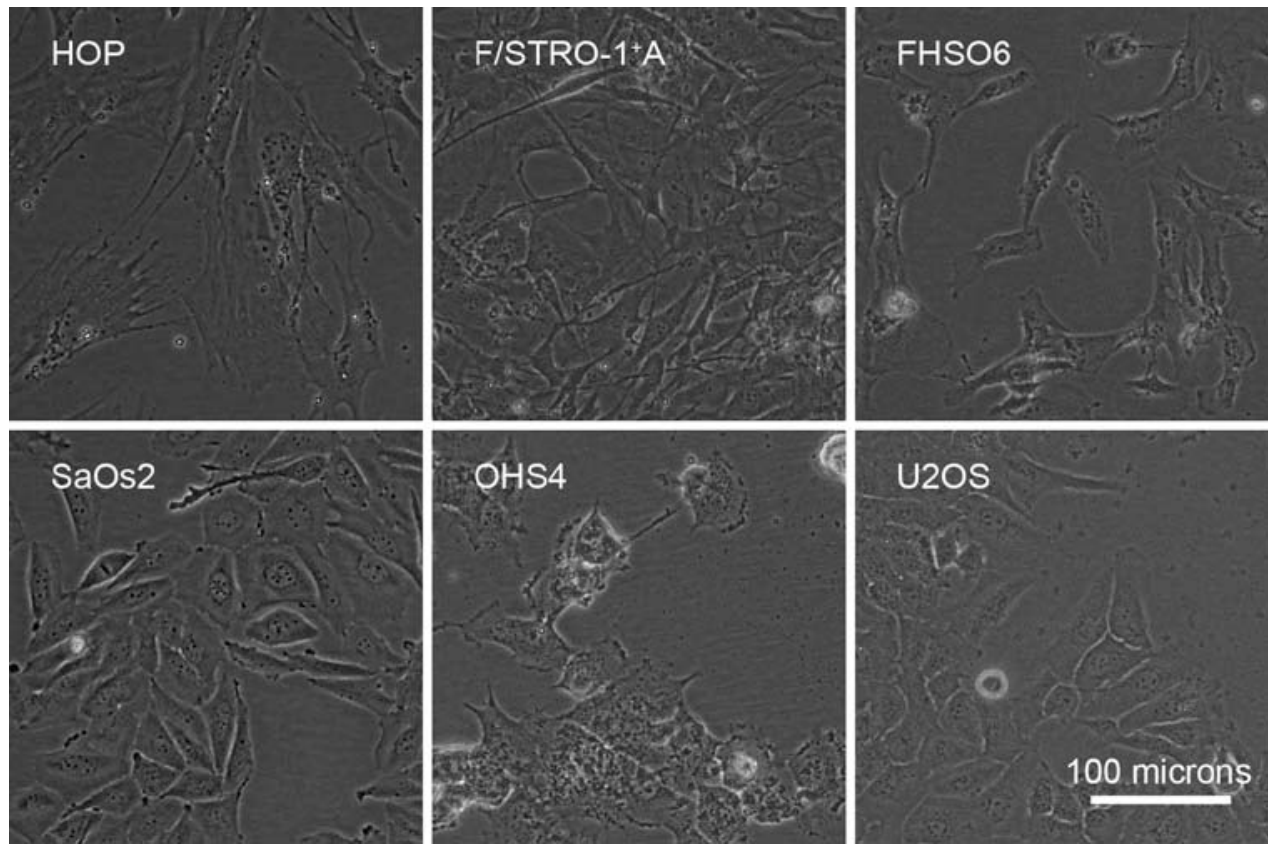

Fig. 2 Immortalized and healthy cells are not affected by the pillars to the same extent as the cancerous cells are. Healthy cells, cells from two immortalized cell lines (top) and three cancerous cell lines (bottom) were grown on micropillared surfaces for $96 \mathrm{~h}$. The cancerous cells show deformation of the entire cell whereas the immortalized cells show small deformations, and the healthy cells rarely show any deformation. The $\mathrm{F} /$ STRO $1^{+}$A cells present deformation at the edges of the cytoskeleton, in particular at the end of filopodia (arrows). The FHSO6 cells show slight indentations over the entire cell and occasionally a deformed nucleus
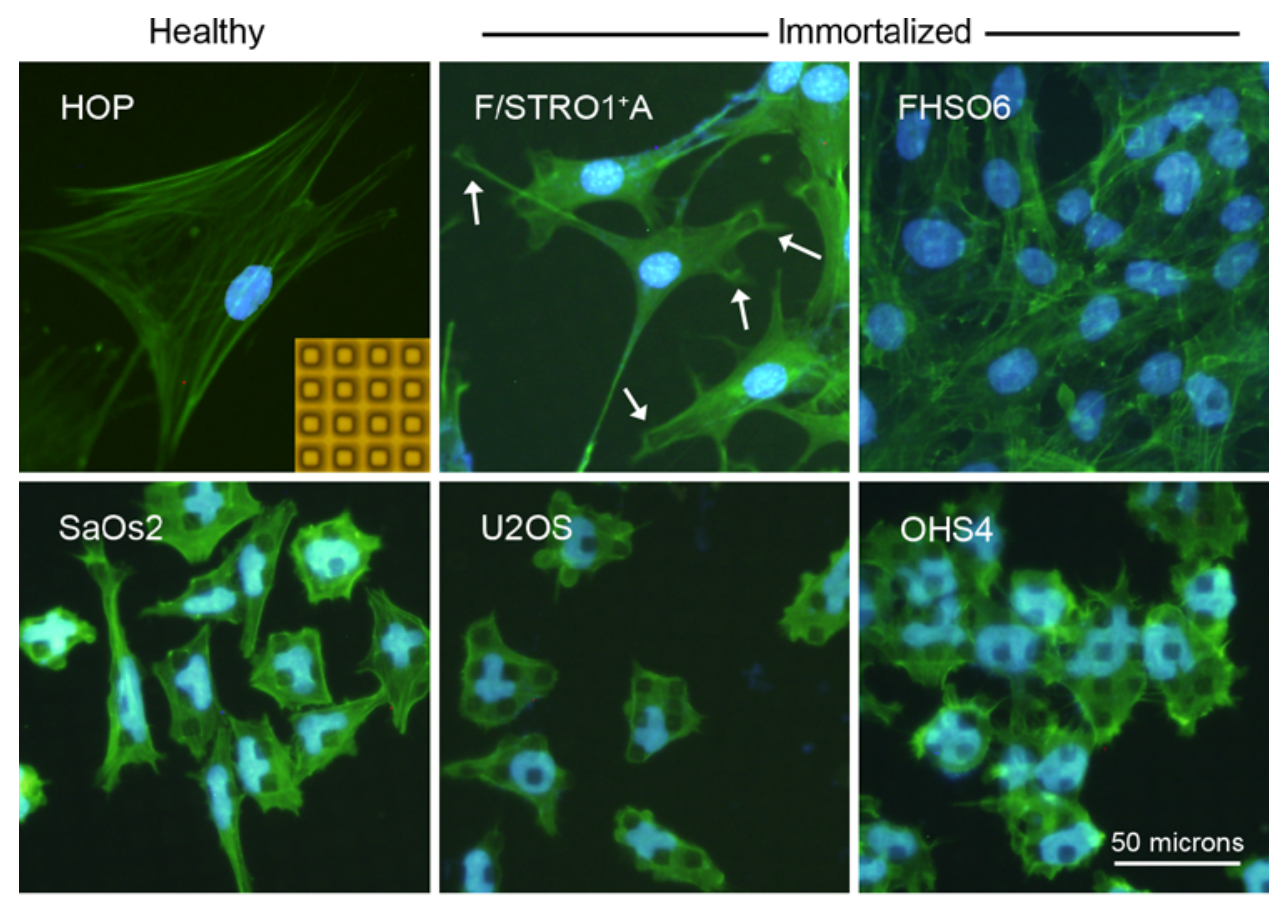

Cancerous 
the pillars can be easily deduced from the immunofluorescence images. The cytoskeleton staining is visible mostly in between the pillars, indicating that there are actin filaments that span the space between the pillars. Additionally, the staining in between the pillars is uniform, indicating a diffuse cytoskeletal arrangement made up of thin disorganized fibers rather than stress fibers.

The immortalized cell lines showed interaction with the surfaces, but not in the same manner as the cancerous cell lines. The F/STRO ${ }^{+}$A cells did not show any deformation of the nucleus at all. In each cell the nucleus was perfectly round and undisturbed. The cytoskeleton was unaffected in the areas around the nucleus, but the extremities of the cytoskeleton were often deformed, as if micropillars had been used by the cell as anchor points. The cells also presented long protrusions (filopodia) that were often terminated by "lasso" shapes (Fig. 2, arrows).

The second immortalized cell line, the FHSO6 cells, were deformed on the pillars, although not as extensively as the cancerous cells. The entire cell appeared to be lightly imprinted with the shape of the pillars. The nucleus of the cells were round and slightly affected by the pillars, except in a few cases where the nucleus was deformed and inserted in between the pillars, as in the cancerous cells.

\subsection{Deformation at short times}

SaOs-2 cells have been shown to deform at delays as short as $6 \mathrm{~h}$ [9] (Fig. 3). At this time point, the cells are not yet well-spread, each cell only extends over one or two pillars. In spite of this the deformation is already clearly visible. With increasing time the cells spread and the deformation increases. On the other hand, at $6 \mathrm{~h}$ the HOP cells are already spread on the surface and most of the cells (about $70 \%$ ) show some deformation. This was visible in the nuclei as a diminution of the fluorescence across the top of the pillars, though most still had a roughly round shape. The cytoskeletons also showed some deformation. Some cells had filopodia that ended on micropillars (Fig. 3) and some had filopodia that ran along the top of a row of
Fig. 3 HOP cells deform at short time points and lose their deformation at longer delays. After being grown on micropillars for $6 \mathrm{~h}$ (top) the cells show deformation of the entire cell, the shape of the pillar is visible in the shape of the cytoskeleton (green or grey) and the nucleus (blue or light grey). At later time points (middle and bottom) the cells show less deformation and there are fewer deformed cells. Cytoskeletal fibers are visible as thin filaments. The cytoskeleton is often seen to interact with the pillars as the edges of the cell: filopodia end on the top of pillars $(6 \mathrm{~h}$, left and $12 \mathrm{~h}$, left $)$ and the shape of the pillar is visible through the cytoskeleton (48 h, right). The SaOs-2 cells show deformation at short time points $(6 \mathrm{~h})$. With increasing time the cells show increasing deformation of the cytoskeleton and of the nucleus (12 h). After several days, the nucleus is completely deformed and has adopted the shape of the space in between the pillars (48 h). (Color figure online)

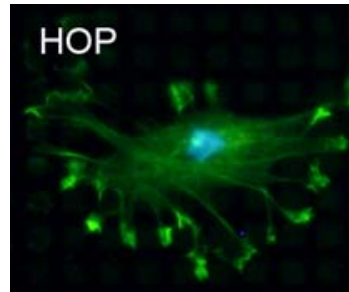

$6 \mathrm{~h}$
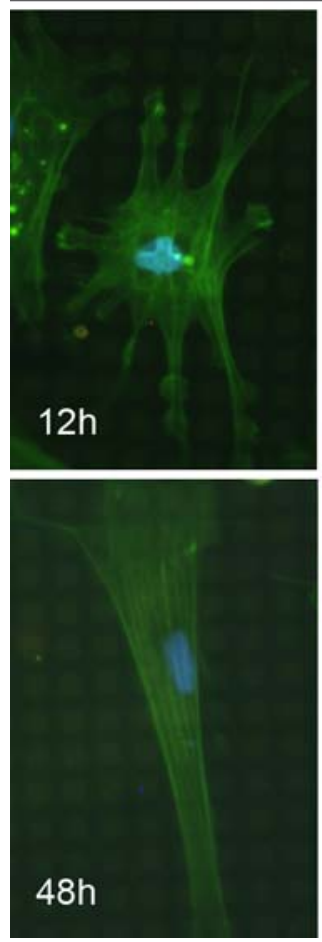
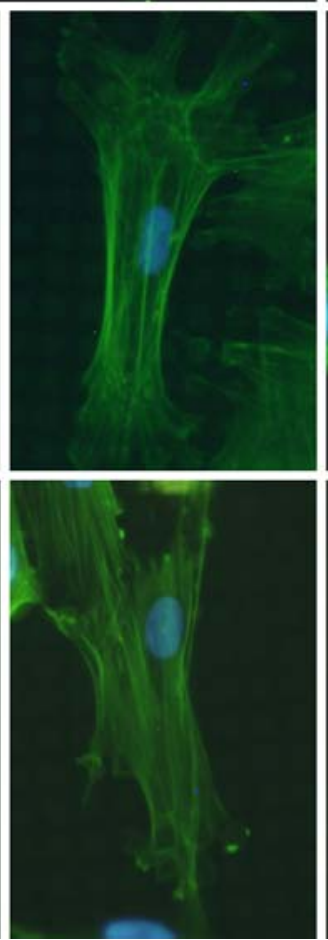

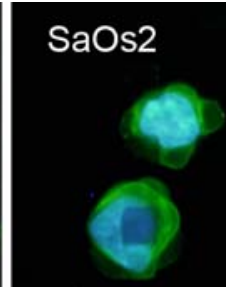

$6 h$
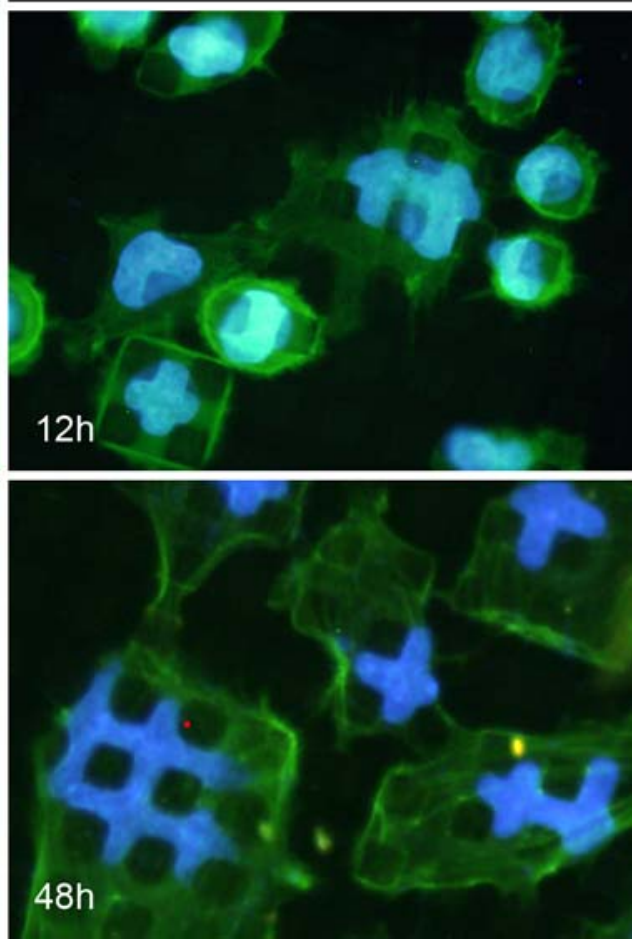
micropillars, or along the trough in between a row of micropillars. The cells whose filopodia ran along the bottom of the interspaces were the most deformed.

At $12 \mathrm{~h}$ the HOP cells have a less rounded, more polarized shape and have spread more on the surface of the pillars to take up a larger area. The fraction of cells showing deformation has decreased (about 50\%) and the deformation of the nucleus is less obvious: the nuclei of the cells are still partially deformed, but less so and appear rounder. On the other hand, all of the SaOs-2 cells show deformation of the cell body and the nucleus. This trend continues in the samples at the later time points and at $48 \mathrm{~h}$ only about $10-20 \%$ of HOP cells have deformed nuclei whereas all of the SaOs- 2 cells are deformed and the extent of nucleus deformation has increased. The deformation in the HOP cells is not extensive: the nuclei have round shapes and the deformation is only visible as a slight loss of fluorescence over the top of the pillars. The cytoskeleton of the HOP cells at later time points have distinct actin fibers. In the SaOs- 2 cells the cytoskeleton staining is diffuse and indicates a disorganized arrangement of thin fibers, rather than the thick fiber bundles present in the case of the HOP cells. This suggests that the cytoskeleton of the HOP cells is more organized than the cytoskeleton of the SaOs-2 cells.

\section{Discussion}

\subsection{The deformation of the cells is due} to the cytoskeleton

Micropatterned surfaces are used to study cell mechanics, but in the literature the cells seeded on these surfaces are often shown to span the surface structures. In our studies, when cancerous cells are grown on micropillars the entire cell deforms and the nucleus in particular adopts a severely distorted shape. Immortalized cells show some deformation as do healthy cells but these lose this deformation at longer times. This is in agreement with data in the literature that suggests that cancerous cells are more deformable than healthy cells [13]. Studies that compare the deformability of cells often use transformed cells to establish trends, in which they show that immortalized cells are also more deformable than their healthy counterparts [15]. At this scale, gravity does not play a great enough role to induce such strong deformations of the nucleus. It is therefore likely that this effect is due to forces generated by the cell. The most likely candidate is the cytoskeleton, which provides structural integrity to the cell and allows movement, both of the cell and of entities inside the cell. Spontaneous deformation has not yet been described and it is surprising that cellular mechanisms would exist that would encourage this type of behavior. By which mechanism do cancerous cells deform to adopt the shape of the pillars? How is the cell able to reorganize its inner structure in response to the surface topography?

To answer these questions we need to better understand the cytoskeleton and the way it may induce such a deformation. The cytoskeleton forms an interconnected network around the cell nucleus [20]. It is connected to the cell wall at the focal contacts and to the nucleus through the LINC complex and lamins [21]. In the area close to the cell membrane a layer of actin filaments forms the actin cortex. Based on this knowledge we propose two possible mechanisms to explain how the deformation of the nucleus inside the cell might occur. Both these mechanisms use the same actors (the cytoskeleton and its linkages) and it is likely that both are occurring simultaneously. In the first mechanism proposed in Fig. 4 we indicate how the forces occurring during cell spreading may affect the interior of the cell. As the cell spreads, a force is exerted outwards, followed by a contraction of the cytoskeleton [2]. On a flat surface this would result in the flattening of the nucleus that would go from a spherical shape in suspension to an lentoid shape when attached to the surface. On a micropillared surface this may result in a downwards force on the cell nucleus, either indirectly because of the stretching of the cell, or directly because of the cortical cytoskeleton filaments that stretch above the nucleus and contract, exerting a force on the interior of the cell. This mechanism would result in the nucleus being pushed downwards in between the pillars as the cell spreads on the surface (Fig. 4a).

The second proposed mechanism involves the pulling down of the nucleus rather than the pushing down from above (Fig. 4b). When the cell attaches to the surface it may form attachment sites to the edges and sides of the pillars. As the cell nucleus is connected to the edges of the cell through the cytoskeleton, when the cell wall attaches to the sides of the pillars the nucleus may be pulled down with

\section{(a) Compression from above}

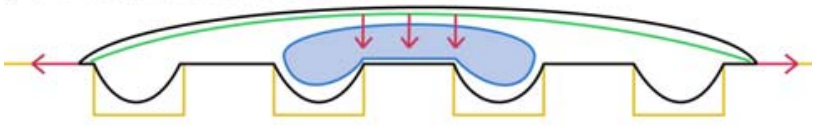

(b) Pull-down from below

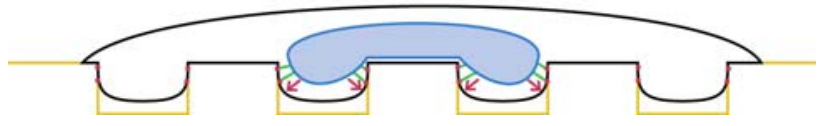

Fig. 4 The nucleus is deformed by forces exerted by the cytoskeleton. Proposed mechanisms are shown in which the nucleus is being pushed down through the spreading forces exerted on the cell (a), and the nucleus is pulled down through the focal point-cytoskeletonnucleus network (b). The cytoskeleton fibers are shown in green (light grey). The arrows represent the force exerted by the cytoskeletal fibers shown. (Color figure online) 
it [20]. It has been shown that unadhered cells in suspension have a cytoskeleton scaffold in place [15], accounting for deformation of the nucleus before the cell has spread over the surface (Fig. 3). In the microscopy images of the actin cytoskeleton it is visible that there is cytoskeleton formation in between the pillars: the area in between the pillars is more stained than the area across the top of the pillars in deformed cells (Fig. 2). This is an indication that there is a cytoskeleton network that is in place in between the pillars, lending credence to the second proposed mechanism.

Studies have tried to explain the balance of forces within the cells. In the tensegrity model mechanical changes in the environment (stresses) are transmitted to the cell through the cytoskeleton which consists of pre-stressed fibers at equilibrium [10]. Thus, in our system, the equilibrium state of the cytoskeleton of cancerous cells would be found after deformation between the pillars. This could indicate that the equilibrium state of cells on the micropillared surface is not the same as on a flat surface and stresses could be transferred differently in these systems.

Many studies have been published recently in which the shape of biological objects has been related to the forces exerted upon them [22]. In the same manner, the shape of the nucleus should procure clues about the forces that are exerted upon it and hence, the architecture of the cell that contains it. A preliminary finding is that the nucleus of cancerous cells on micropillars is under constant stress and has plastic properties: its deformation increases steadily with time [9]. Additional data on the properties of the nucleus-cytoskeleton scaffold should be found in experiments in which cytoskeleton disruptors are used. Modification of the shape of the nucleus upon release of the cytoskeletal pressure should confirm that the cytoskeleton is an active actor in the deformation of the cell and demonstrate the viscoelastic properties of the nucleus. Additionally, the use of specific disruptors should provide information on which filaments are responsible and the amount of force they can generate.

\subsection{The deformation is greatly influenced}

by the biology of the cells

As we have shown, cancerous cells, immortalized cells and healthy cells have varying degrees of responsiveness to microstructured surfaces. This is most likely due to the difference in biology of the three cell types.

Generally immortalized cells are infected with the SV40 virus which blocks the p53 and pRB tumour suppressor genes [14]. These genes help regulate the cell cycle, and, in particular, stimulation of p53 was found to be associated with an increase in organized microfilament bundles [23]. When blocked, these can no longer prevent rapid proliferation, as is the case with cancerous cells. The transformed cells in our study were found to not be tumorigenic. They were chosen for their osseous phenotype and their size, which is comparable to SaOs-2. The F/STRO $1^{+} \mathrm{A}$ cells displayed features of immature osteoprogenitor cells [17] and FHSO6 cells appear to express characteristics of immediate precursors of mature osteoblast-like cells [18]. Nevertheless, important differences have been found in the cytoskeletons of immortalized cells compared to their healthy counterparts. Cells immortalized with SV40, in which p53 and pRB have been suppressed, have been reported to produce less actin and tubulin than normal cells [13], and a less ordered cytoskeleton [15]. It is interesting to note that the modification on only one gene can induce such a difference, whereas cancerous cells have many gene modification compared to healthy cells. The cells used in this study and the previous study have different p53 and pRB statuses: SaOs-2 expresses neither, MG-63 only expressed pRB and U2OS expresses both [24]. Despite the discrepancy in gene modification, all of the cancerous cells used behaved similarly, indicating that $\mathrm{pRB}$ and p53 are not solely responsible for the deformation and that other genes must be altered as well. This change in the cytoskeletal properties may also be an indirect consequence of the rapid proliferation of the cells, which would result in decreased cytoskeleton-building periods.

Cancerous cell lines come from cells that have been harvested from tumours. The cells used in this study all came from osteosarcoma. Cancerous cells have a modified cytoskeleton which allows them to be motile and replicate quickly. They are also known to be more deformable than healthy cells [13]. Moreover, this has been related to their metastatic potential: cells that are more likely to form tumours in vivo are more deformable [15, 25]. Yet, the deformability is not necessarily related to the rigidity of the cells: studies have shown that the rigidity of adhered HOP cells is intermediate between that of SaOs-2 and MG-63 cells, two osteosarcoma cell lines [26], which have both been shown by us to deform on micropillared substrates [9]. Hence, the deformation is related to the inner forces within the cell more than the rigidity of the cell. It is likely that cancerous cells are more deformable simply because of their role: in order to form metastases, the cells need to deform sufficiently to pass through tissue.

It is interesting to note that the deformation is universal in the case of cancerous cells, whereas the effect in immortalized cells differs. HOP and F/STRO- ${ }^{+}$A cells have similar features when placed on flat surfaces (Fig. 1). They are elongated and have a lot of filopodia-like protrusions, indicating that they have very active cytoskeletal networks. In the immunohistochemistry images (Figs. 2 and 3), HOP, F/STRO1 ${ }^{+} \mathrm{A}$ and FHSO6 show distinct actin fibers whereas the cytoskeleton of the cancerous cells is 
more uniform across the whole cell, indicating less organization into fiber bundles. These observations are in accordance with reports that the cytoskeleton of cancerous cells is less organized than healthy cells [13]. The organization present in the non-malignant cells seems to be able to prevent severe deformation of the cell and its nucleus. The cytoskeletal filaments may be able to surround the nucleus and shield it from external topography. In this way, healthy cells may have less flexibility in their shape, whereas cancerous cells adapt to the surface on which they grow, enabling them to survive on surfaces other than their native tissue. Hence, cancerous cells may be able to survive in other parts of the body and under higher stress than healthy cells.

The behaviour of the cells at short times may also provide clues regarding the early organization of the cytoskeleton. Upon adhesion to a substrate, cells initially form attachment points within a matter of minutes. This is followed by a phase of cytoskeleton filament formation that occurs within hours of attachment. At longer time scales (hours to days) the cell begins to exert forces on its surroundings and migrate on the surface. In cancerous cells, deformation is visible as soon as the cells adhere to the surface [9] and is maintained with time. This indicates that the cytoskeleton acts on the nucleus very early on. In the case of the HOP cells, deformation is also visible at short timepoints, during the initial stages of spreading, but is gradually lost with time as the cell continues to spread (Fig. 2). By contrast, the cancerous cells show increased deformation with time. This indicates a disparity in behaviour: the healthy cells oppose deformation, whereas the cancerous cells promote it. The entire cell, including the nucleus, is under continuous pressure to deform. The deformation in the case of the HOP cells is most likely to be due to the proposed pull-down mechanism rather than the pushing from above during spreading as increased spreading results in lesser deformation.

\section{Conclusion}

We have confirmed that upon adhesion to a micro-pillared substrate cancerous cells can undergo extensive deformation. It was found that this deformation also occurs in healthy cells, but only during the initial stages of adhesion. Limited deformation was also seen in immortalized cells. The entire spectrum of biological transformations that immortalized cells undergo is not yet well understood. Because these deformations are not the same as the deformations that healthy or cancerous cells undergo we can postulate that immortalization also induces a change in the cytoskeleton. Micropillared surfaces have shown their utility in identifying changes in phenotype and could be used as tools to distinguish between cancerous and noncancerous cells. Additionally, this surface-induced deformation must induce stress within the cells. This behaviour could provide novel and quantitative information on how stress may affect the performance of the cells (differentiation, proliferation) as well as facilitating a deeper knowledge of the intracellular organization and the mechanics of the cytoskeleton.

Acknowledgments Financial support provided through the European Community's "Marie Curie Actions" under contract MRTNCT-2004-005516 (BioPolySurf). V. H. also acknowledges the support of TUBITAK (the Scientific and Technical Research Council of Turkey) through the project Nanobiomat (TBAG 105T508). We also acknowledge Prof. A. Aydinli of Bilkent University for the patterned templates.

\section{References}

1. Su WT, Chu IM, Yang JY, Lin CD. The geometric pattern of a pillared substrate influences the cell-process distribution and shapes of fibroblasts. Micron. 2006;37:699-706.

2. Tan JL, Tien J, Pirone DM, Gray DS, Bhadriraju K, Chen CS. Cells lying on a bed of microneedles: an approach to isolate mechanical force. Proc Natl Acad Sci. 2003;100:1484-9.

3. Steinberg T, Schultz S, Spatz JP, Grabe N, Mussig E, Kohl A, et al. Early keratinocyte differentiation on micropillar interfaces. Nano Lett. 2007;7:287-94.

4. Thery M, Racine V, Piel M, Pépin A, Dimitrov A, Chen Y, et al. Anisotropy of cell adhesive microenvironment governs cell internal organization and orientation of polarity. PNAS. 2006;103:19771-6.

5. Bischofs IB, Schmidt SS, Schwarz US. Effect of adhesion geometry and rigidity on cellular force distributions. Phys Rev Lett. 2009;103:048101.

6. Anselme K, Bigerelle M, Noël B, Loison I, Hardouin P. Kinetic study of the expression of $\beta$-catenin, actin and vinculin during osteoblastic adhesion on grooved titanium substrates. Biomed Mater Eng. 2004;14:545-56.

7. Tsai WB, Ting YC, Yang JY, Lai JY, Liu HL. Fibronectin modulates the morphology of osteoblast-like cells (MG-63) on nano-grooved substrates. J Mater Sci Mater Med. 2009;20:136778.

8. Dalby MJ, Riehle MO, Yarwood SJ, Wilkinson CD, Curtis AS. Nucleus alignment and cell signaling in fibroblasts: response to a micro-grooved topography. Exp Cell Res. 2003;284:274-82.

9. Davidson P, Özçelik H, Hasirci V, Reiter G, Anselme K. Microstructured surfaces cause severe but non-detrimental deformation of the cell nucleus. Adv Mater. 2009;21:3586-90.

10. Ingber DE. Tensegrity: the architectural basis of cellular mechanotransduction. Annu Rev Physiol. 1997;59:575-99.

11. Taddei A, Hediger F, Neumann FR, Gasser SM. The function of nuclear architecture: a genetic approach. Annu Rev Genet. 2004;38:305-45.

12. Reddy KL, Zullo JM, Bertolino E, Singh H. Transcriptional repression mediated by repositioning of genes to the nuclear lamina. Nature. 2008;452:243-7.

13. Ben-Ze'ev A. The cytoskeleton in cancer cells. Biochim Biophys Acta. 1985;780:197-212.

14. Ali SH, DeCaprio JA. Cellular transformation by SV40 large T antigen: interaction with host proteins. Semin Cancer Biol. 2001;11:15-23. 
15. Guck J, Schinkinger S, Lincoln B, Wottawah F, Ebert S, Romeyke M, et al. Optical deformability as an inherent cell marker for testing malignant transformation and metastatic competence. Biophys J. 2005;88:3689-98.

16. Anselme K, Broux O, Noël B, Bouxin B, Bascoulergue G, Dudermel A-F, et al. In vitro control of human bone marrow stromal cells for bone tissue engineering. Tissue Eng. 2002;8: 941-53.

17. Oyajobi BO, Lomri A, Hott M, Marie PJ. Isolation and characterization of human clonogenic osteoblast progenitors immunoselected from fetal bone marrow stroma using STRO-1 monoclonal antibody. J Bone Miner Res. 1999;14:351-61.

18. Fromigue O, Kheddoumi N, Lomri A, Marie PJ, Body JJ. Breast cancer cells release factors that induced apoptosis in human bone marrow stromal cells. J Bone Miner Res. 2001;16: $1600-10$.

19. Rodan GA, Majeska RJ. Phenotypic maturation of osteoblastic osteosarcoma cells in culture. Prog Clin Biol Res. 1982;110 Pt B:249-59.

20. Bidwell JP, Alvarez M, Feister H, Onyia J, Hock J. Nuclear matrix proteins and osteoblast gene expression. J Bone Miner Res. 1998;13:155-67.
21. Wang N, Tytell JD, Ingber DE. Mechanotransduction at a distance: mechanically coupling the extracellular matrix with the nucleus. Nat Rev Mol Cell Biol. 2009;10:75-82.

22. Frolov VA, Zimmerberg J. Shaping biological matter. Nat Mater. 2009;8:173-4.

23. Comer KA, Dennis PA, Armstrong L, Catino JJ, Kastan MB, Kumar CC. Human smooth muscle alpha-actin gene is a transcriptional target of the p53 tumor suppressor protein. Oncogene. 1998;16:1299-308.

24. Heessen S, Leonchiks A, Issaeva N, Sharipo A, Selivanova G, Masucci MG, et al. Functional p53 chimeras containing the Epstein-Barr virus Gly-Ala repeat are protected from Mdm2- and HPV-E6-induced proteolysis. Proc Natl Acad Sci U S A. 2002; 99:1532-7.

25. Ochalek T, Nordt FJ, Tullberg K, Burger MM. Correlation between cell deformability and metastatic potential in B16-F1 melanoma cell variants. Cancer Res. 1988;48:5124-8.

26. Docheva D, Padula D, Popov C, Mutschler W, Clausen-Schaumann H, Schieker M. Researching into the cellular shape, volume and elasticity of mesenchymal stem cells, osteoblasts and osteosarcoma cells by atomic force microscopy. J Cell Mol Med. 2008;12:537-52. 